Article

\title{
Electron Transfer at Gold Nanostar Assemblies: A Study of Shape Stability and Surface Density Influence
}

\section{Mariana Chirea}

Chemistry and Biochemistry Department, Faculty of Sciences, Universidade do Porto, Rua Campo Alegre 687, 4169-007, Porto, Portugal; E-Mail: mariana.chirea@fc.up.pt;

Tel.: +351-220-402-612; Fax: +351-220-402-659

Received: 17 January 2013; in revised form: 25 February 2013 / Accepted: 26 February 2013 / Published: 12 March 2013

Abstract: Gold nanostars of $\sim 70 \mathrm{~nm}$ tip to tip distances were synthesized by a seed mediated method and covalently self-assembled on 1,5-pentanedithiol modified electrodes. Electron transfer kinetics at the AuNS/dithiol modified electrodes were studied as a function of AuNS surface density which was varied by increasing their self-assembly time from $8 \mathrm{~h}, 16 \mathrm{~h}, 24 \mathrm{~h}$ to $32 \mathrm{~h}$. Excellent electrocatalytic properties of AuNSs were observed toward electrochemistry of $\left[\mathrm{Fe}(\mathrm{CN})_{6}\right]^{4-/ 3-}$ redox couple. The apparent heterogeneous electron transfer constant, $k_{\text {et }}$, has progressively increased with the surface density of AuNSs bonded to the electrodes from $0.65 \times 10^{-5} \mathrm{~cm} \mathrm{~s}^{-1}(8 \mathrm{~h}), 1.47 \times 10^{-5} \mathrm{~cm} \mathrm{~s}^{-1}(16 \mathrm{~h})$, $3.95 \times 10^{-5} \mathrm{~cm} \mathrm{~s}^{-1}(24 \mathrm{~h})$ to an excellent $85.0 \times 10^{-5} \mathrm{~cm} \mathrm{~s}^{-1}(32 \mathrm{~h})$. Electrochemical charging of nanostars was confirmed, for the first time, by 79 times increase of double layer capacitance, $C_{\mathrm{dl}}$, from $0.34 \mu \mathrm{F}(8 \mathrm{~h})$ to $27 \mu \mathrm{F}(32 \mathrm{~h})$. The electrochemical charging of AuNSs had also a strong influence on the electron tunneling process through the 1,5PDT molecules being more efficient at dense layers of AuNSs. The tunneling parameter, $\beta$, has decreased from $1.13 \AA^{-1}(16 \mathrm{~h})$ to $0.50 \AA^{-1}(32 \mathrm{~h})$. The AuNSs were chemically stable toward $\left[\mathrm{Fe}(\mathrm{CN})_{6}\right]^{4-/ 3-}$ showing no change in shape after electrochemical measurements.

Keywords: molecular electronics; gold nanostars; electron transfer; electrochemical charging 


\section{Introduction}

Nowadays, molecular nanoelectronics represent and important research field in which organic molecules and inorganic nanomaterials are combined to form electronic components [1-17]. For this purpose, self-assembled monolayers (SAMs) of bifunctional organic thiols have been extensively used for the chemical binding of metallic nanomaterials to various substrates and studies of electron transport [1-7]. The bifunctional organic thiols are good molecular candidates to be used for surface modifications and as linkers because of their easy preparation, high stability and ordered structures [8-17]. Among the bifunctional organic thiols, dithiols form densely packed and highly ordered films with an upright molecular structure when self-assembled on gold or platinum surfaces [8-17]. These ordered structures of dithiol SAMs are dependent of chain length and dithiols concentrations in alcohols. For example, Qu et al. [9] have demonstrated that 1,6-hexanedithiol self-assembled on gold substrate from ethanolic solution have formed highly ordered and stable films as compared to its corresponding monothiol, C6SH SAMs, this stability being evidenced by a negative shift in reductive peak potential from monothiol SAMs to dithiol SAMs. Within 1,6-hexanedithiol SAMs, two types of sulfur species, i.e., thiolate-type sulfur (Au-SR) and tail group thiol sulfur (RSH), were identified by XPS measurements indicating the stand-up dithiol configuration in the SAMs [9]. The same upright structure of the dithiol molecules covalently anchored to gold substrates was identified by FT-IR measurements. ${ }^{4}$ For this short chain dithiol, a concentration of $15 \mathrm{mM}$ or lower was enough to yield extremely ordered films [4,9]. Akkerman et al. [10] have demonstrated that in order to obtain an almost full standing-up phase of 1,14-tetradecanedithiol (C14) a concentration of $30 \mathrm{mM}$ of dithiol in ethanol was required. At intermediate $(3 \mathrm{mM})$ and low concentrations $(0.3 \mathrm{mM})$, few looped molecules (the chain of the dithiol is anchored to the surface with both sulphur atoms) or predominant looped molecules in the 1,14-tetradecanedithiol SAM were observed [10]. Nonresonant electron tunneling was shown to be the electrical transport mechanism through these dithiol SAMs with the current density decreasing exponentially with increasing molecule length (or tunneling-barrier width) [15]. Few reports have been published on studies concerning the use of aliphatic chain dithiols as linkers for metallic nanoparticles to study charge transfer [3,4,16,17].

In this work, 1,5-pentanedithiol (1,5PDT) was used for anchoring gold nanostars (AuNSs) to electrodes. The gold nanostars are multibranched nanoparticles with multiple plasmon resonances of which the lower energy ones, corresponding to the nanostar tips and core-tip interactions, are the most sensitive to environmental changes $[18,19]$. For this reason, many research reports have been focused on studies concerning optical properties and optical sensing applications of gold nanostars [18-24]. For example, Rodríguez-Lorenzo et al. [21] have found an overall $10^{10}$ SERS enhancement factor at bilayers of mixed thiols (1,5-naphtalenedithiol and 1-octanethiol) and gold nanostars as compared to the bilayers of the same mixed dithiols and spherical gold nanoparticles deposited on optically thick Au substrates. Dondapati et al. [24] have detected streptadivin molecules upon binding to individual biotin-modified gold nanostars by shifts in the plasmon resonance. They have found that concentrations as low as $0.1 \mathrm{nM}$ of streptadivin produce a shift of the tip related plasmon resonances of about $2.3 \mathrm{~nm}$ corresponding to $5.3 \mathrm{meV}$ [24]. The electrocatalytic properties of gold nanostars could be also strongly influenced by their sensitivity to environmental changes. Their unusual shape could bring 
different contributions to the electron transfer processes due to different sizes existent in their structure.

The purpose of the present work was to find out: (i) if nanostars could produce different responses in an electrochemical system than previously studied nanomaterials and (ii) if their size and shape were preserved during the electrochemical process studied. The morphology of gold nanostars and their crystallographic features were analyzed by transmission electron microscopy (TEM). Their optical properties were analyzed by UV-Vis spectroscopy. Surface coverage and morphologies of gold nanostars self-assembled on 1,5PDT modified electrodes before and after electrochemical measurements were analyzed by scanning electron microscopy (SEM). Charge transfer at the 1,5PDT/AuNS modified electrodes was studied experimentally by cyclic voltammetry, square wave voltammetry and electrochemical impedance spectroscopy (EIS) in aqueous solution of $0.0005 \mathrm{M}$ $\left[\mathrm{Fe}(\mathrm{CN})_{6}\right]^{4-/ 3-}$ and $0.1 \mathrm{M} \mathrm{NaClO}_{4}$. There is substantial experimental evidence demonstrating that the kinetics of electron transfer at AuNS modified electrodes was dependent on the surface density of gold nanostars and time of reaction. Fittings of EIS data have revealed electrochemical charging of AuNS together with an efficient electron transfer process. SEM analyses of electrodes surface before and after electrochemical measurements have revealed the preservation of AuNSs' morphology.

\section{Results and Discussion}

The average core diameters and tip length of gold nanostars synthesized following Liz-Marzán method [18] were: $49.72 \pm 0.10 \mathrm{~nm}$ and $19.44 \pm 0.35 \mathrm{~nm}$ (Figure 1a). The gold nanostars displayed two surface plasmon bands, one of them being localized at the tips and producing a dominant broad feature at the edge of the visible range $(837 \mathrm{~nm}$, Figure $1 \mathrm{~b})$. The cores produce a surface plasmon band at shorter wavelength $(545 \mathrm{~nm})$.

Figure 1. (a) TEM image of gold nanostars. Scale bar: $200 \mathrm{~nm}$. Inset: digital image of AuNSs' solution; (b) Optical spectrum of gold nanostars.
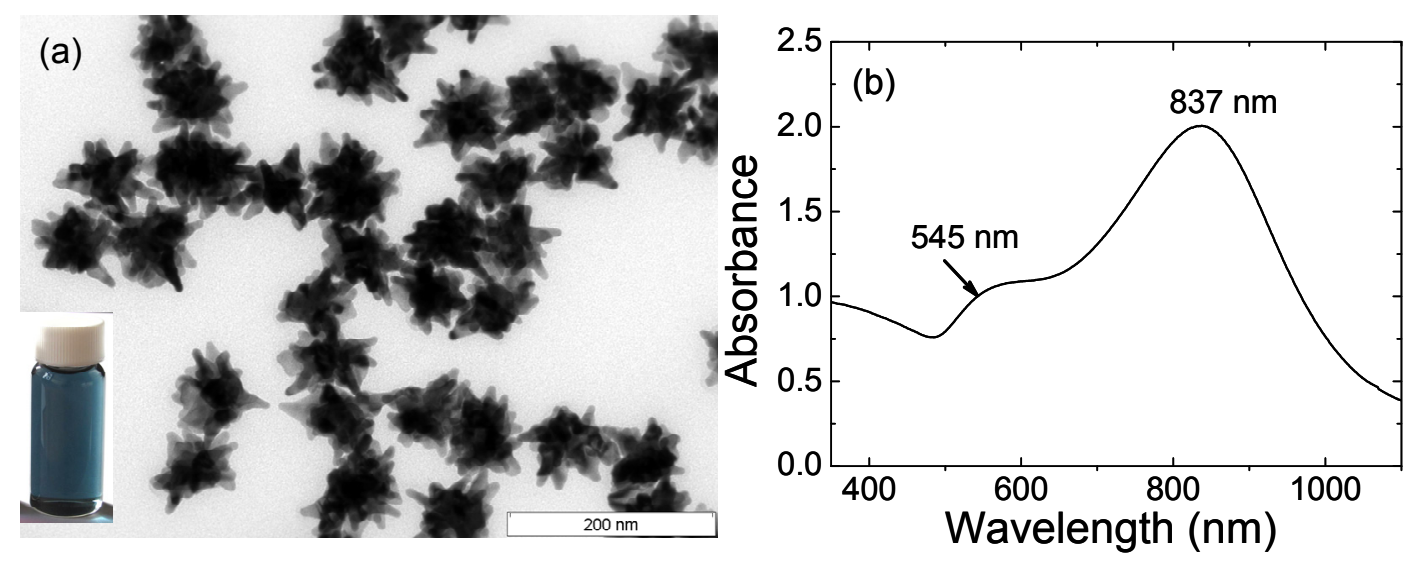


\subsection{Electrochemistry of $\left[\mathrm{Fe}(\mathrm{CN})_{6}\right]^{4-/ 3-}$ at Au-1,5PDT-AuNS Modified Electrodes for AuNSs'} Self-Assembly Time of $8 \mathrm{~h}$

The gold nanostars were covalently self-assembled on gold electrodes using 1,5PDT as a cross linker molecule. It is important to emphasize that the purification of gold nanostars represent an important step for their self-assembly on dithiol modified electrodes. Repeated washing of gold nanostars with ethanol and Millipore water followed by one washing step in $\mathrm{H}_{2} \mathrm{SO}_{4}: \mathrm{H}_{2} \mathrm{O}_{2}=1: 1$ and consecutive washing with Millipore water allows a partial removal of PVP from their surface [25] and makes possible their covalent self-assembly on Au-1,5PDT-SAM modified electrodes. The structure of the 1,5PDT SAM was expected to be very similar to the structure of 1,6hexanedithiol SAM [9] because there is only one $-\mathrm{CH}_{2}$ - difference between the chemical structures of the two dithiols. A dense coverage of the 1,5PDT SAM with up-right structure was achieved upon self-assembly of the dithiol from $15 \mathrm{mM}$ ethanolic solution [3,4,9]. The covalent binding of gold nanostars to dithiol films was observed to take place at the tips of the nanostars [21]. Bilayers with increased AuNSs' surface coverage were fabricated by immersing the 1,5PDT modified electrodes in aqueous solution of AuNSs (1 $\mathrm{mg} / \mathrm{mL}$ ) and let to react at room temperature for $8 \mathrm{~h}, 16 \mathrm{~h}, 24 \mathrm{~h}$ and $32 \mathrm{~h}$ under slow magnetic stirring. As mentioned previously, the first experimented self-assembly time was $8 \mathrm{~h}$. The surface coverage with AuNSs was low (Figure $2 \mathrm{a}-\mathrm{c}$ ) but the electron transfer process at this particular electrode was very interesting.

In the CVs a strong symmetric flow of Faradaic currents was observed upon consecutive scans in $0.0005 \mathrm{M}\left[\mathrm{Fe}(\mathrm{CN})_{6}\right]^{3-/ 4-}$ aqueous solution (Figure $2 \mathrm{~d}-\mathrm{f}$ ), with a transition from an irreversible to a reversible electrochemical behavior. SEM analysis of this electrode before and after electrochemical measurements (Figure 2a-c) has revealed similar surface coverage and no evident change in shape of gold nanostars. The first 10 cyclic voltammograms show high peak-to-peak potential separations and low current densities (for example the 10th $\mathrm{CV}$ in Figure $2 \mathrm{~d}$ has a $\Delta E \mathrm{p}=0.653 \mathrm{~V}$ and $j_{\mathrm{a}}=1.47 \mu \mathrm{A} \mathrm{cm}^{-2} v s$. $\mathrm{Ag} / \mathrm{AgCl}$ ). Consecutive measurements of $\mathrm{CVs}$ (Figure 2e,f) show a progressive and continuous increase of peak current densities and continuous decrease of peak-to-peak potential separation indicating that the interfacial electron transfer was becoming faster. The final cyclic voltammogram has reached a peak-to-peak potential separation of $\Delta E \mathrm{p}=0.108 \mathrm{~V}$ at a current density of $j_{\mathrm{a}}=3.45 \mu \mathrm{A} \mathrm{cm}^{-2}$ (red curve in Figure 2f,g) as compared to bare gold for which $\Delta E \mathrm{p}=0.088 \mathrm{~V}$ and $j=3.60 \mu \mathrm{A} \mathrm{cm}^{-2}$ (black dashed curve in Figure $2 \mathrm{~g}$ ) illustrating slightly slower electron transfer (ET) kinetics at the AuNSs modified electrode ( $8 \mathrm{~h}$ self-assembly time). In the square wave voltammograms one can observe current densities of $j_{\mathrm{a}}=7.1 \mu \mathrm{A} \mathrm{cm} \mathrm{cm}^{-2}(0.233 \mathrm{~V})$ at Au-1,5PDT-AuNS modified electrode $(8 \mathrm{~h}$, red curve in Figure $2 \mathrm{~h}$ ) as compared to bare gold for which the current density was $j_{\mathrm{a}}=10 \mu \mathrm{A} \mathrm{cm} \mathrm{cm}^{-2}$ at $0.233 \mathrm{~V}$ (black dashed curve in Figure 2h). Electrochemical impedance spectroscopy measurements were performed in order to obtain a detailed characterization of the interfacial properties of the modified electrodes. The modeling of EIS spectra for bare gold and AuNS modified electrodes' surface was done using Randles electrical equivalent circuit (Figure 3a) or a second more complex electrical equivalent circuit (Figure 3b) which consist of a solution resistance Rs connected in series with the film capacitance and resistance, $C_{\mathrm{f}}$ and $R_{\mathrm{f}}$ (elements in parallel to each other) and in series with the double layer capacitance $C_{\mathrm{dl}}$, charge transfer resistance, $R_{\mathrm{CT}}$ and Warburg impedance $\left(Z_{\mathrm{w}}\right)$ respectively (Figure $3 \mathrm{~b}$ ). The complex impedance is the sum of the real, $Z^{\prime}$, and 
imaginary, $Z$ ", components that originate mainly from elements of resistance and capacitance of the cell, respectively. The capacitance and resistance of the film, $C_{\mathrm{f}}$ and $R_{\mathrm{f}}$ give information about film area [26,27] and electron tunneling efficiency through the dithiol layer [3,4,28,29].

The double layer capacitance is dependent on the conductive (will increase) or insulating (will decrease) properties of the interface (Table 1), whereas the charge transfer resistance is due to electron transfer generated by the redox probes present in the electrolyte solution. The fittings of all Nyquist diagrams were done by tentatively approaching the experimental curves through appropriate choices of the parameter values. All fittings were checked by evaluation of the chi-squared parameter, which was of $10^{-4}$ order of magnitude yielding $98 \%$ confidence bound between parameters. One important parameter for further evaluation of the electrochemical features of the modified electrodes is the charge transfer resistance. The charge-transfer resistance is inverse proportional to the exchange current under equilibrium $I_{0}(1)$ :

$$
R C T=\frac{R T}{n F I 0}
$$

and can be used to evaluate the experimental heterogeneous electron-transfer rate constant, $k_{\mathrm{et}}$, based on the Equations (1) and (2):

$$
I_{0}={ }_{n F A k e t}[c]
$$

where $R$ is the gas constant, $T$ is the temperature $(\mathrm{K}), A$ is the electrode area $\left(0.0314 \mathrm{~cm}^{2}\right),[c]$ corresponds to the bulk concentration of the redox probe $\left(0.0005 \mathrm{~mol} \mathrm{~cm}^{-3}\right)$, and $n$ is the number of transferred electrons per molecule of the redox probe $(n=1)$ for the $\left[\mathrm{Fe}(\mathrm{CN})_{6}\right]^{3-/ 4-}$ probes.

Figure 2. (a-c) SEM images with different magnifications of top view of AuNS modified electrode for $8 \mathrm{~h}$. Scale bars: $10 \mu \mathrm{m}$ (a), $5 \mu \mathrm{m}(\mathbf{b})$ and $1 \mu \mathrm{m}(\mathbf{c})$; (d-f) Consecutive CVs measured at the Au-1,5PDT-AuNS modified electrode in aqueous solution of $0.0005 \mathrm{M}$ $\left[\mathrm{Fe}(\mathrm{CN})_{6}\right]^{4-/ 3-}$ and $0.1 \mathrm{M} \mathrm{NaClO}_{4}$. Scan rate $50 \mathrm{mV} / \mathrm{s}$; $(\mathbf{g}-\mathbf{h})$ Comparison of final cyclic voltammograms (g), square wave voltammograms (h, sw amplitude $50 \mathrm{mV}$, frequency $10 \mathrm{~Hz}$ and potential step $2 \mathrm{mV}$ ) and Nyquist diagrams (i, frequency range $10 \mathrm{kHz}$ to $0.1 \mathrm{~Hz}$, amplitude $10 \mathrm{mV}$ ) measured at Au-1,5PDT-AuNS modified electrodes (red curves), bare gold (black dashed curves) and Au-1,5PDT-SAM (blue curves) in $0.0005 \mathrm{M}\left[\mathrm{Fe}(\mathrm{CN})_{6}\right]^{4-/ 3-}$ and $0.1 \mathrm{M} \mathrm{NaClO}_{4}$.
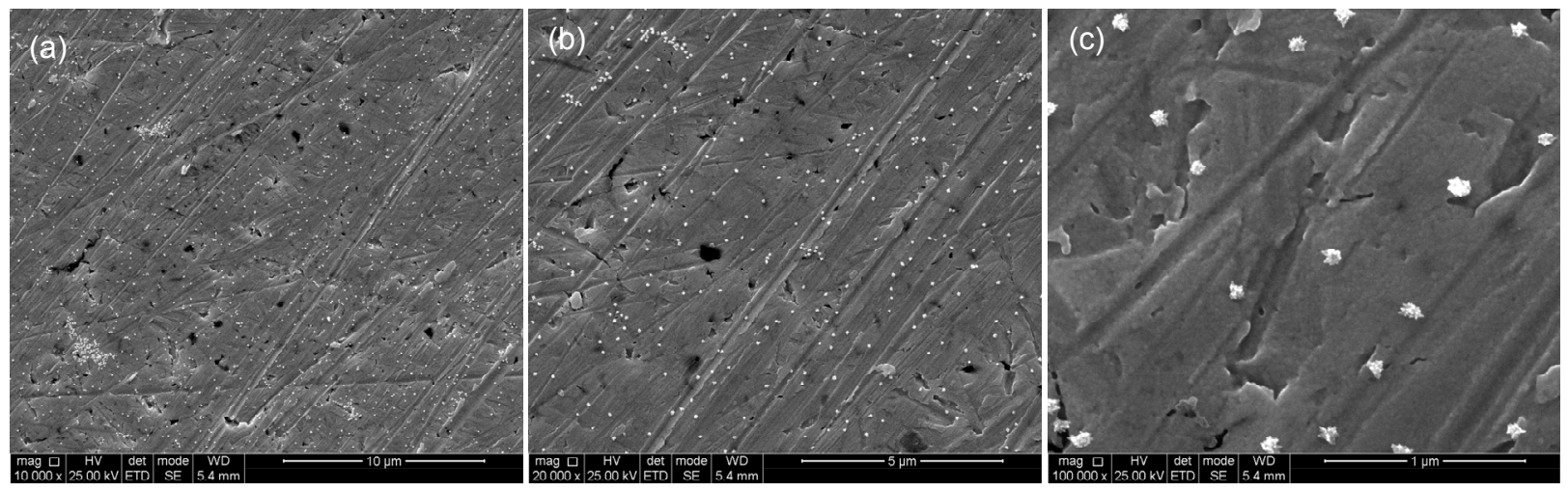
Figure 2. Cont.
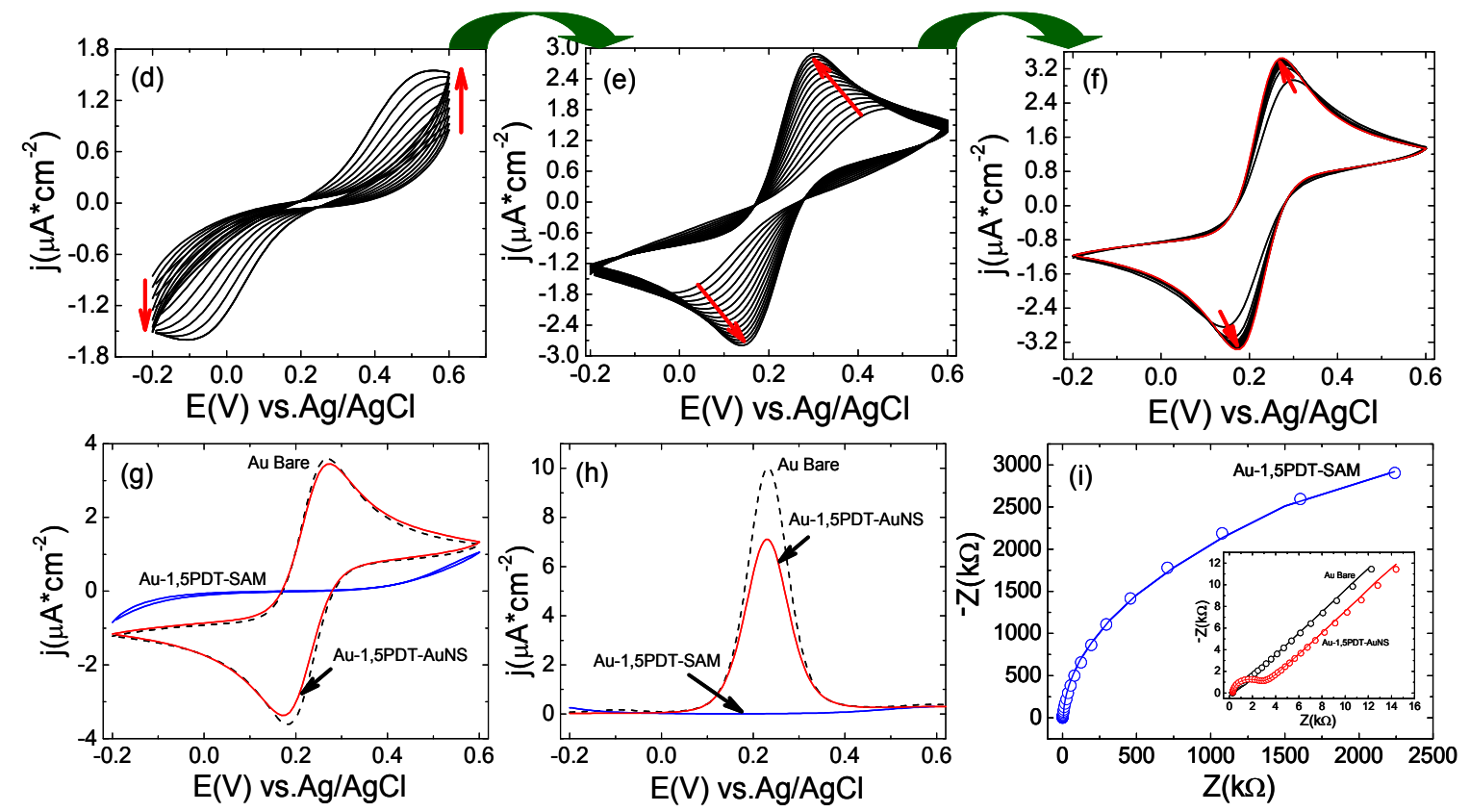

Figure 3. Randles circuit (a) and the electrical equivalent circuit utilized to model impedance spectra at film modified electrodes (b).

(a)
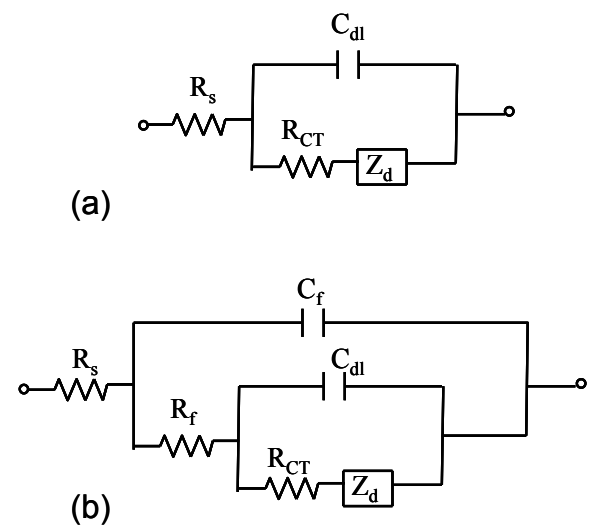

Table 1. Parameter values obtained from fittings of the impedance spectra represented in Figure $2 \mathrm{i}$ using non-linear square fit procedure ${ }^{\dagger}$.

\begin{tabular}{llccll}
\hline EIS plot & $\boldsymbol{R}_{\mathbf{s}}(\mathbf{k} \boldsymbol{\Omega})$ & $\boldsymbol{C}_{\mathbf{f}}(\boldsymbol{\mu} \mathbf{F})$ & $\boldsymbol{R}_{\mathbf{f}}(\mathbf{k} \boldsymbol{\Omega})$ & $\boldsymbol{C}_{\text {dl }}(\boldsymbol{\mu} \mathbf{F})$ & $\boldsymbol{R}_{\mathrm{CT}}(\mathbf{k} \boldsymbol{\Omega})$ \\
\hline Au Bare & 0.284 & - & - & 1.89 & 0.61 \\
& $(0.70)$ & & & $(0.11)$ & $(0.97)$ \\
Au-1,5PDT-SAM & 3.52 & 0.252 & 2558.4 & 0.28 & 2027 \\
& $(0.60)$ & $(0.48)$ & $(0.26)$ & $(0.92)$ & $(0.97)$ \\
Au-1,5PDT-AuNS & 0.303 & - & - & 0.34 & 2.60 \\
$(8 \mathrm{~h})$ & $(0.72)$ & & & $(0.98)$ & $(0.85)$ \\
\hline
\end{tabular}

${ }^{\dagger}$ The percent errors from the fit of each element are given in parenthesis. 
Table 2. Heterogeneous electron transfer rate constants estimated using $R_{\mathrm{CT}}$ values presented in Table 1 and based on Equations (1) and (2).

\begin{tabular}{ll}
\hline EIS plot & $\boldsymbol{k}_{\text {et }}\left(\mathbf{c m ~ s}^{-1}\right)$ \\
\hline Au bare & $2.78 \times 10^{-5}$ \\
Au-1,5PDT-SAM & $0.84 \times 10^{-8}$ \\
Au-1,5PDT-AuNS & $0.65 \times 10^{-5}$ \\
$(8 \mathrm{~h})$ & \\
\hline
\end{tabular}

The estimated heterogeneous electron transfer rate constants for bare gold, Au-1,5PDT-SAM and Au-1,5PDT-AuNS $(8 \mathrm{~h})$ modified electrodes are presented in Table 2. The $k_{\text {et }}$ at 1,5PDT modified electrode was three orders of magnitude smaller than at bare gold electrode indicating a strong hindered electron transfer. The electrochemical insulating nature of the 1,5PDT monolayer is illustrated also by the increase of solution resistance, $R_{\mathrm{s}}$ (Table 1). The fact that the tunneling resistance behaves as a solution resistance was previously demonstrated by Diao et al. [28,29].

The estimated electron tunneling coefficient $\beta$ was $0.97 \AA^{-1}$ for a tunneling distance of $8.75 \AA$ ( $7 \times 1.25 \AA$ SAM chain length) $[3,4]$. This $\beta$ value is much smaller than that of $1.3-1.8 \AA^{-1}$ estimated for through space tunneling [30] indicating that the electron tunneling at the Au-1,5PDTAuNS modified electrodes is through-bond tunneling. The heterogeneous electron transfer rate constant, $k_{\text {et }}$, at AuNS modified electrode was smaller than that at bare gold electrode (Table 2) illustrating a slower electron transfer kinetics at the Au-1,5PDT-AuNS modified electrode being in accordance with lower current densities in the CVs and SQWVs (Figure 2g,h) than those at bare gold electrode. This is due to low surface coverage with AuNSs and probably due to their large size (49.72 $\mathrm{nm}$ average core diameters and $19.44 \mathrm{~nm}$ average tip lengths). After our knowledge, there are no studies published on the electrochemical properties of gold nanostars. Only recently, we have published a report in which we have presented a comparison of kinetics of electron transfer at two different self-assembled films: one containing dithiol and larger gold nanostars, the other containing the same dithiol and spherical nanoparticles [31]. A 2.5 times increase of double layer capacitance was observed at $45 \%$ surface coverage with gold nanostars. The study shows for the first time, a possible shape effect of gold nanostars in an electrochemical process as compared to spherical nanoparticles [31]. The 1,5pentanedithiol self-assembled on gold electrodes has greatly hindered the heterogeneous electron transfer between the $\left[\mathrm{Fe}(\mathrm{CN})_{6}\right]^{3-/ 4-}$ redox probes in solution and the underlying bare gold electrode, this electronic blocking being illustrates by the disappearance of Faradaic currents in the cyclic voltammograms (maximum 50 scans) and square wave voltammograms (blue curves in Figure $2 \mathrm{~g}, \mathrm{~h}$ ) and increase of solution resistance (Table 1). The corresponding Nyquist plot in Figure 2i (blue EIS plot) shows a depressed semicircle for all range of frequencies $(10,000 \mathrm{~Hz}$ to $0.1 \mathrm{~Hz})$ with no evident diffusion profile at low frequencies which corresponds to a high resistance to the charge transfer and a three order magnitude decrease of the heterogeneous electron transfer constant (see parameters values for Au-1,5PDT-SAM in Tables 1 and 2). The strong insulating behavior of 1,5PDT-SAM is almost nullified by the AuNSs consecutively attached to the electrodes with low surface coverage, generating EIS plots with small semicircles at high and intermediated frequencies and well defined diffusion profiles at low frequencies demonstrating semi-infinite planar diffusion to the film (slope was unity for the red EIS plot in inset of Figure 2i). The switching-on of $\left[\mathrm{Fe}(\mathrm{CN})_{6}\right]^{3-/ 4-}$ electrochemistry upon AuNS 
self-assembly suggest that the rate limiting step is the exchange of electrons between redox probes in solution and AuNS at the electrode/solution interface and not the electron tunneling through the dithiol layer. Compared to spherical gold nanoparticles (AuNPs) or gold nanorods (AuNRs) self-assembled on electrodes using as cross linker molecules similar dithiols [3,4,31], the gold nanostars have revealed a more complex electrochemical behavior. For example, at low surface coverage with larger AuNSs, the electrochemistry of $\left[\mathrm{Fe}(\mathrm{CN})_{6}\right]^{4-/ 3-}$ was recovered completely (Figure 2b, [31]), whereas at quit high surface coverage with spherical gold nanoparticles (13 $\mathrm{nm}$ average diameters) the electrochemical process is far from being reversible (Figure 2f,g, [31]). In both cases the 1,5PDT was used as cross-linker molecule and its strong insulating electronic behavior could not be nullified by the spherical gold nanoparticles but it was nullified by the gold nanostars [31]. Moreover, the heterogeneous electron transfer constant was smaller at Au-1,5PDTAuNP $\left(0.087 \times 10^{-5} \mathrm{~cm} \mathrm{~s}^{-1}\right)$ than at Au-1,5PDT-AuNS $\left(3.04 \times 10^{-5} \mathrm{~cm} \mathrm{~s}^{-1}\right)$ modified electrodes [31].

\subsection{Electrochemistry of $\left[\mathrm{Fe}(\mathrm{CN})_{6}\right]^{4-/ 3-}$ at Au-1,5PDT-AuNS Modified Electrodes for AuNSS, Self-Assembly Time of $16 \mathrm{~h}$}

The fabrication of 1,5PDT-AuNSs films with slightly higher surface coverage of AuNSs was achieved by increasing their self-assembly time to $16 \mathrm{~h}$. In Figure $4 \mathrm{a}-\mathrm{c}$ are presented the corresponding SEM images at different magnification, whereas Figure 4d-e illustrate the evolution of consecutive cyclic voltammetry, square wave voltammetry and electrochemical impedance measurements performed at the Au-1,5PDT-AuNSs modified electrode (16 h) in aqueous solution of $0.0005 \mathrm{M}\left[\mathrm{Fe}(\mathrm{CN})_{6}\right]^{4-/ 3-}$ and $0.1 \mathrm{M} \mathrm{NaClO}_{4}$. As previously observed, there is a transition from slow kinetics to fast kinetics of electron transfer. Both cyclic and square wave voltammograms show a continuous change from high (wine and green curves in Figure 4d,e) to small (cyan and red curves in Figure 4e,d) peak-to-peak potential separations and a continuous increase of current densities. In this case, the transition takes place with higher electrochemical steps and is faster than at the previous modified electrode (higher $k_{\text {et }}$, Table 3 ) because the surface loading with AuNSs is higher. In the cyclic voltammograms (Figure 5d) the peak-to peak potential separation and current densities varied as follows: $\Delta E \mathrm{p}=0.225 \mathrm{~V}, j_{\mathrm{a}}=2.64 \mu \mathrm{A} \mathrm{cm} \mathrm{cm}^{-2}$ (wine curve), $\Delta E \mathrm{p}=0.144 \mathrm{~V}, j_{\mathrm{a}}=3.28 \mu \mathrm{A} \mathrm{cm}{ }^{-2}$ (green curve), $\Delta E \mathrm{p}=0.108 \mathrm{~V}, j_{\mathrm{a}}=3.56 \mu \mathrm{A} \mathrm{cm} \mathrm{cm}^{-2}$ (blue curve), $\Delta E \mathrm{p}=0.094 \mathrm{~V}, j_{\mathrm{a}}=3.70 \mu \mathrm{A} \mathrm{cm}^{-2}$ (magenta curve), $\Delta E \mathrm{p}=0.091 \mathrm{~V}, j_{\mathrm{a}}=3.76 \mu \mathrm{A} \mathrm{cm}^{-2}$ (cyan curve), $\Delta E \mathrm{p}=0.090 \mathrm{~V}, j_{\mathrm{a}}=3.80 \mu \mathrm{A} \mathrm{cm}^{-2}$ (dark cyan curve), and $\Delta E \mathrm{p}=0.090 \mathrm{~V}, j_{\mathrm{a}}=3.82 \mu \mathrm{A} \mathrm{cm} \mathrm{cm}^{-2}$ (red curve) as compared to bare gold for which $\Delta E \mathrm{p}=0.088 \mathrm{~V}, j_{\mathrm{a}}=3.60 \mu \mathrm{A} \mathrm{cm} \mathrm{cm}^{-2}$.

Keeping in mind that the concentration of redox probes in solution is constant $(0.0005 \mathrm{M})$, the variation of these parameters illustrates an electrocatalytic effect of AuNSs. This electrocatalytic effect is even more evident in the SQWVs (Figure 4e) which show a drastic increase of net Faradaic currents, i.e., $j_{\mathrm{a}}=5.80 \mu \mathrm{A} \mathrm{cm} \mathrm{c}^{-2}$ at $0.244 \mathrm{~V}$ (wine curve), $j_{\mathrm{a}}=10.64 \mu \mathrm{A} \mathrm{cm}^{-2}$ at $0.232 \mathrm{~V}$ (green curve), $j_{\mathrm{a}}=14.65 \mu \mathrm{A} \mathrm{cm}^{-2}$ at $0.227 \mathrm{~V}$ (blue curve), $j_{\mathrm{a}}=17.47 \mu \mathrm{A} \mathrm{cm}^{-2}$ at $0.227 \mathrm{~V}$ (pink curve), $j_{\mathrm{a}}=19.00 \mu \mathrm{A} \mathrm{cm}^{-2}$ at $0.224 \mathrm{~V}$ (cyan curve), $j_{\mathrm{a}}=20.20 \mu \mathrm{A} \mathrm{cm} \mathrm{cm}^{-2}$ at $0.224 \mathrm{~V}$ (dark cyan curve), $j_{\mathrm{a}}=20.65 \mu \mathrm{A} \mathrm{cm}^{-2}$ at 0.224 $\mathrm{V}$ (red curve) as compared to bare gold for which $j_{\mathrm{a}}=10 \mu \mathrm{A} \mathrm{cm} \mathrm{cm}^{-2}$ at $0.230 \mathrm{~V}$ (Figure $2 \mathrm{~h}$, black dashed curve). It is important to note that the impedance is decreasing at any given frequency for consecutive measurements at the Au-1,5PDT-AuNS modified electrode (16 h, Figure 4f) in aqueous solution of 
$0.0005 \mathrm{M}\left[\mathrm{Fe}(\mathrm{CN})_{6}\right]^{4-/ 3-}$ and $0.1 \mathrm{M} \mathrm{NaClO}_{4}$. The decrease of impedance values was observed for the first time at AuNP/polymer multilayer modified electrodes [26,27]. This decrease of EIS values was achieved by increasing the number of AuNPs layers electrostatically self-assembled on gold electrodes using positively charged polymers [26,27]. In recent reports we have demonstrated that the impedance values could also decrease if small nanorods (AuNRs) were covalently attached to electrodes [3] or if, despite their size, the AuNRs surface density was increased [4]. In this work, a monodisperse sample of gold nanostars was used for modifying the electrodes, being varied only their surface density. As presented in Figures 2 and 4, the impedance values were decreasing from $8 \mathrm{~h}$ to $16 \mathrm{~h}$ self-assembly time of AuNSs, so again, by increasing the surface density of AuNSs. What does differ from the other electrochemical systems is the fact that the EIS values were decreasing also during consecutive measurements performed at the same Au-1,5PDT-AuNS modified electrodes in aqueous solution of $0.0005 \mathrm{M}\left[\mathrm{Fe}(\mathrm{CN})_{6}\right]^{4-/ 3-}$ for both low and high surface coverage with AuNSs. This reflects a different electrocatalytic behavior of gold nanostars as compared to their spherical or nanorod counterparts [3,4,26,27,29]. Fittings of EIS plots (in Figure 4f) show a continuous decrease of apparent charge transfer resistance, $R_{\mathrm{CT}}$ (Table 3 ) in parallel with a continuous increase of double layer capacitance, $C_{\mathrm{dl}}$. The variation of these two parameters proves a continuous improvement of electron transfer kinetics and, simultaneously, charges accumulation (!) at the Au-1,5PDT-AuNSs modified electrode surface $(16 \mathrm{~h})$. The charge accumulation on the AuNS layer can arise from two contributions: slow electron transfer at the gold nanostar surface due to their large sizes and electronic blocking generated by the underlying 1,5pentanedithiol layer. These synergic influences could explain the progressive transition from CVs with large peak-to-peak separation and low peak current densities to $\mathrm{CVs}$ with narrow peak-to-peak separation and high peak current densities as presented in Figure $2 \mathrm{~d}-\mathrm{f}$ and Figure 4 d,e. The capacitance of the film, $C_{\mathrm{f}}$, was almost constant (Table 3 ) proving that the area of the electrode does not change during the electrochemical process. The constant value of this parameter confirms the stability of nanostar shape and size during the electrochemical process as observed in SEM images (Figure 4a-c). Rodriguez-Lorenzo et al. [32] have observed that gold nanostars can undergo chemical reshaping in the presence of cetyltrimethylammonium bromide (CTAB) after $8 \mathrm{~h}$ of reaction. By increasing the $\mathrm{CTAB}$ concentration, the reshaping process was speeded-up (5 h) [32]. Zou et al. have demonstrated that gold nanorods were protected against thermal or chemical reshaping by PVP which has hindered CTAB diffusion to the surface of rods [33]. In our work, if the nanostars were reshaping into smaller nanomaterials, the capacitance of the film, $C_{\mathrm{f}}$, could have decreased illustrating the diminishing of the overall electrode area during the electrochemical process. Contrarily, as presented in Table 3, the $C_{\mathrm{f}}$ was slightly increasing with consecutive electrochemical measurements suggesting only a swelling of AuNS layer upon penetration of redox probes into the film but no reshaping of AuNSs. The variation of this parameter also suggests that the PVP was not removed completely from the surface of AuNSs.

The resistance of the film, which is a tunneling resistance, $R_{\mathrm{f}}$, was progressively decreasing (Table 3 ), demonstrating a progressive improvement of electron tunneling through the aliphatic chains of the 1,5PDT-SAM and toward the underlying bare gold electrode surface. The variation of these electrochemical parameters (Table 3) demonstrates a complex electron transfer process generated by a synergic influence of both electrochemical charging of gold nanostars and electron tunneling through the insulating 1,5PDT layer. 
The large sizes of gold nanostars seem to generate initially a slow electron transfer process, which becomes faster upon continuous electrochemical measurements. In other words, the AuNSs need first to accumulate enough electrons in order to generate a faster electron transfer process. Another cause of the transition from slow to fast electron transfer process during consecutive measurements could be the shape of gold nanostars. By comparing the different sizes existent in a nanostar it may be possible that the ET at the tips of gold nanostars $(19.44 \mathrm{~nm})$ to be faster than ET at their cores $(49.72 \mathrm{~nm})$, this difference of electron transfer kinetics generating charge accumulation at the surface of AuNSs.

Figure 4. (a-c) SEM images recorded after electrochemical measurements performed at Au-1,5PDT-AuNS modified electrodes (16 h self-assembly time for AuNSs). Scale bars: $5 \mu \mathrm{m}(\mathbf{a}), 2 \mu \mathrm{m}$ (b) and $500 \mathrm{~nm}(\mathbf{c}) ;(\mathbf{d}-\mathbf{f})$ Evolution of cyclic (d) and square wave voltammetry (e) and EIS (f) measurements recorded at the Au-1,5PDT-AuNS modified electrode in aqueous solution of $0.0005 \mathrm{M}\left[\mathrm{Fe}(\mathrm{CN})_{6}\right]^{4-/ 3-}$ and $0.1 \mathrm{M} \mathrm{NaClO}_{4}$. Scan rate $50 \mathrm{mV} / \mathrm{s}$. Frequency $10 \mathrm{~Hz}$; (g-i) Comparison of final cyclic (g), square wave voltammograms (h) and EIS spectra (i) recorded in the same conditions at Au-1,5PDT-AuNS modified electrodes (red curves) and bare gold (black dashed curves).
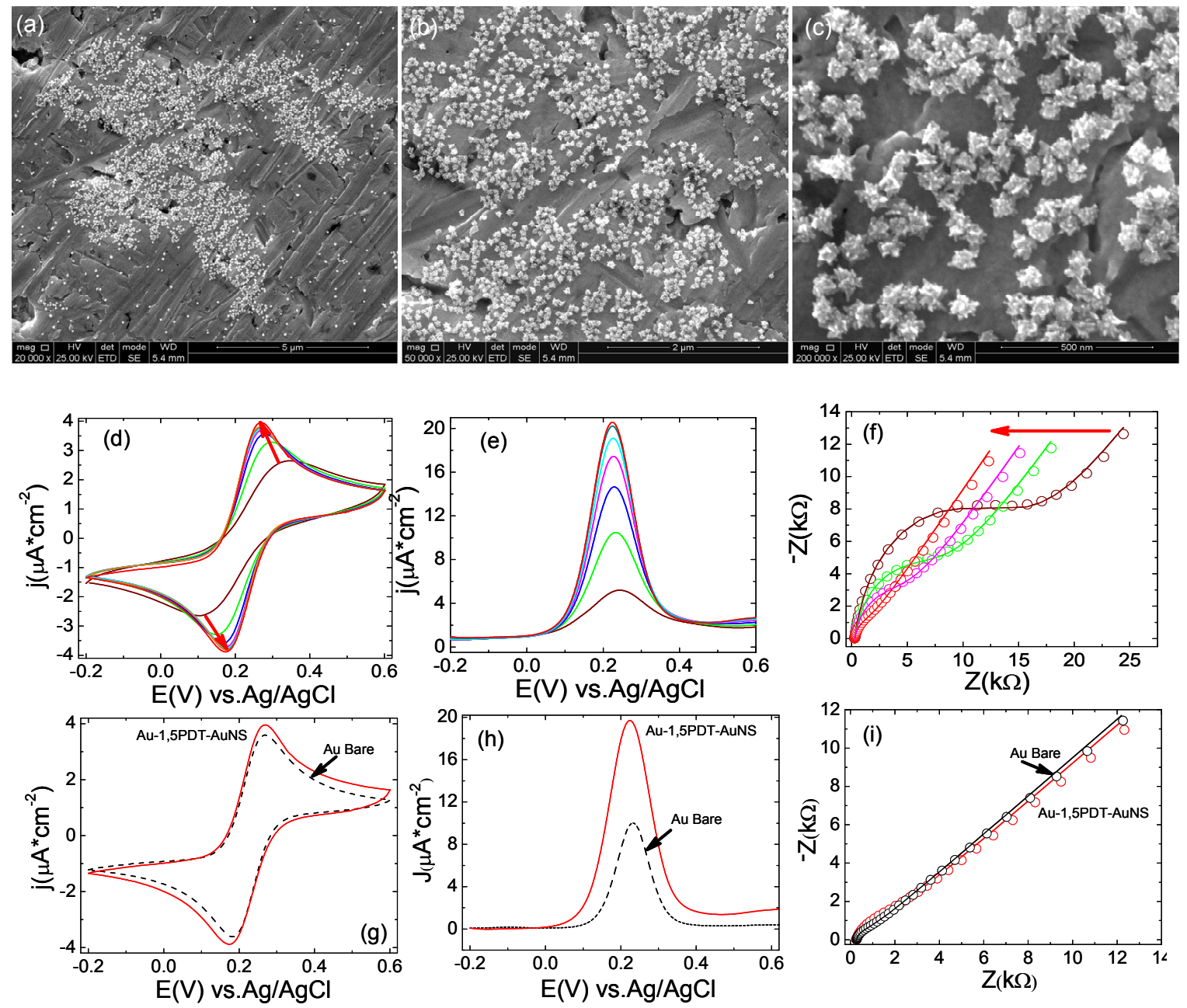
Table 3. Parameters values obtained from fittings of the impedance spectra represented in Figure 4f.

\begin{tabular}{cccccc}
\hline EIS plot & $\boldsymbol{R}_{\mathbf{s}}(\mathbf{k} \boldsymbol{\Omega})$ & $\boldsymbol{C}_{\mathbf{f}}(\boldsymbol{\mu} \mathbf{F})$ & $\boldsymbol{R}_{\mathbf{f}}(\mathbf{k} \boldsymbol{\Omega})$ & $\boldsymbol{C}_{\mathbf{d l}}(\boldsymbol{\mu} \mathbf{F})$ & $\boldsymbol{R}_{\mathbf{C T}}(\mathbf{k} \boldsymbol{\Omega})$ \\
\hline \multirow{2}{*}{ wine } & 0.307 & 2.66 & 4.46 & 1.60 & 9.15 \\
& $(1.50)$ & $(1.21)$ & $(0.92)$ & $(0.79)$ & $(0.45)$ \\
green & 0.312 & 2.70 & 4.90 & 1.69 & 8.80 \\
& $(1.0)$ & $(1.26)$ & $(0.96)$ & $(0.72)$ & $(0.42)$ \\
blue & 0.295 & 2.70 & 2.12 & 1.73 & 4.87 \\
& $(1.26)$ & $(0.92)$ & $(0.96)$ & $(0.63)$ & $(0.93)$ \\
magenta & 0.300 & 2.76 & 2.36 & 1.81 & 4.70 \\
& $(1.06)$ & $(0.69)$ & $(0.94)$ & $(0.65)$ & $(0.94)$ \\
cyan & 0.280 & 2.50 & 0.97 & 1.77 & 3.07 \\
& $(1.10)$ & $(0.91)$ & $(1.25)$ & $(0.58)$ & $(1.12)$ \\
dark cyan & 0.294 & 2.80 & 1.54 & 2.02 & 2.70 \\
& $(1.15)$ & $(0.89)$ & $(0.52)$ & $(0.65)$ & $(0.91)$ \\
red & 0.296 & - & - & 3.18 & 1.15 \\
& $(1.09)$ & & & $(1.35)$ & $(0.87)$ \\
\hline
\end{tabular}

Table 4. Heterogeneous electron transfer constants estimated from fittings of impedance spectra represented in Figure $5 \mathrm{f}$ and the corresponding electron tunneling coefficients, $\beta$.

\begin{tabular}{ccc}
\hline EIS plot & $\boldsymbol{k}_{\text {et }}\left(\mathbf{c m ~ s}^{-\mathbf{1}}\right)$ & $\left.\boldsymbol{\beta} \mathbf{(}^{-\mathbf{1}}\right)$ \\
\hline wine & $0.18 \times 10^{-5}$ & 1.13 \\
green & $0.19 \times 10^{-5}$ & 1.11 \\
blue & $0.35 \times 10^{-5}$ & 1.04 \\
magenta & $0.36 \times 10^{-5}$ & 1.04 \\
cyan & $0.55 \times 10^{-5}$ & 0.99 \\
dark cyan & $0.62 \times 10^{-5}$ & 0.97 \\
red & $1.47 \times 10^{-5}$ & 0.87 \\
\hline
\end{tabular}

Regardless of the cause of this completely new and unusual electrochemical behavior, it is evident that by consecutive electrochemical measurements and increasing the surface density of AuNSs attached to the electrodes, the overall electron transfer process was improved. The heterogeneous electron transfer rate constant has progressively increased from $0.18 \times 10^{-5} \mathrm{~cm} \cdot \mathrm{s}^{-1}$ to $1.47 \times 10^{-5} \mathrm{~cm} \cdot \mathrm{s}^{-1}$ (Table 4) demonstrating a continuous improvement of the overall electron transfer process at the Au1,5PDT-AuNS modified electrode (16 h self-assembly time of AuNSs) with consecutive electrochemical measurements. Moreover, the electron tunneling coefficient, $\beta$, was progressively decreasing (Table 4) demonstrating a continuous and excellent improvement of the electron tunneling process through the underlying 1,5PDT-SAM layer. In previous reports was demonstrated that the electron transfer at nanomaterial modified electrodes was dependent on the size of nanomaterials, number of layers self-assembled on electrodes, presence of pinholes in the films, nature of outermost layer, type of surface bonding and surface density [1,3-6,16,26,27,29]. None of these reports have presented spontaneous charging of electrodes surface during a simple electrochemical process as demonstrated here. Like in a capacitor, it appears that one plate (the redox probes in solution) is 
transferring electrons to the parallel plate (the layer of AuNSs), which is storing them slowly and continuously. At the AuNS layer, part of these electrons was further tunneled through the 1,5PDT layer and underlying bare electrode surface. The presented experiments and modeling of EIS spectra, strongly suggest that the AuNSs could act as energy storage nanodevices [34,35].

\subsection{Electrochemistry of $\left[\mathrm{Fe}(\mathrm{CN})_{6}\right]^{4-/ 3-}$ at Au-1,5PDT-AuNS Modified Electrodes for AuNSs'}

Self-Assembly Time of $24 \mathrm{~h}$

A self-assembly time of $24 \mathrm{~h}$ has yielded 1,5PDT-AuNS films with higher surface coverage of AuNSs. Scanning electron microscopy analysis of the third Au-1,5PDT-AuNS modified electrode (Figure 5a-c) has revealed a dense coverage with AuNSs, some areas of the modified electrode being more covered with AuNSs than other areas (less covered surface areas were marked with white arrows in Figure 5b,c).

Figure 5. (a-c) SEM images and (d-i) electrochemistry of $0.0005 \mathrm{M}\left[\mathrm{Fe}(\mathrm{CN})_{6}\right]^{4-/ 3-}$ at the third Au-1,5PDT-AuNS modified electrode for $24 \mathrm{~h}$ self-assembly time of AuNSs. Scale bars in SEM images were: $10 \mu \mathrm{m}$ (a), $2 \mu \mathrm{m}$ (b) and $500 \mathrm{~nm}$ (c), respectively. The electrochemical conditions are the same as previously presented.
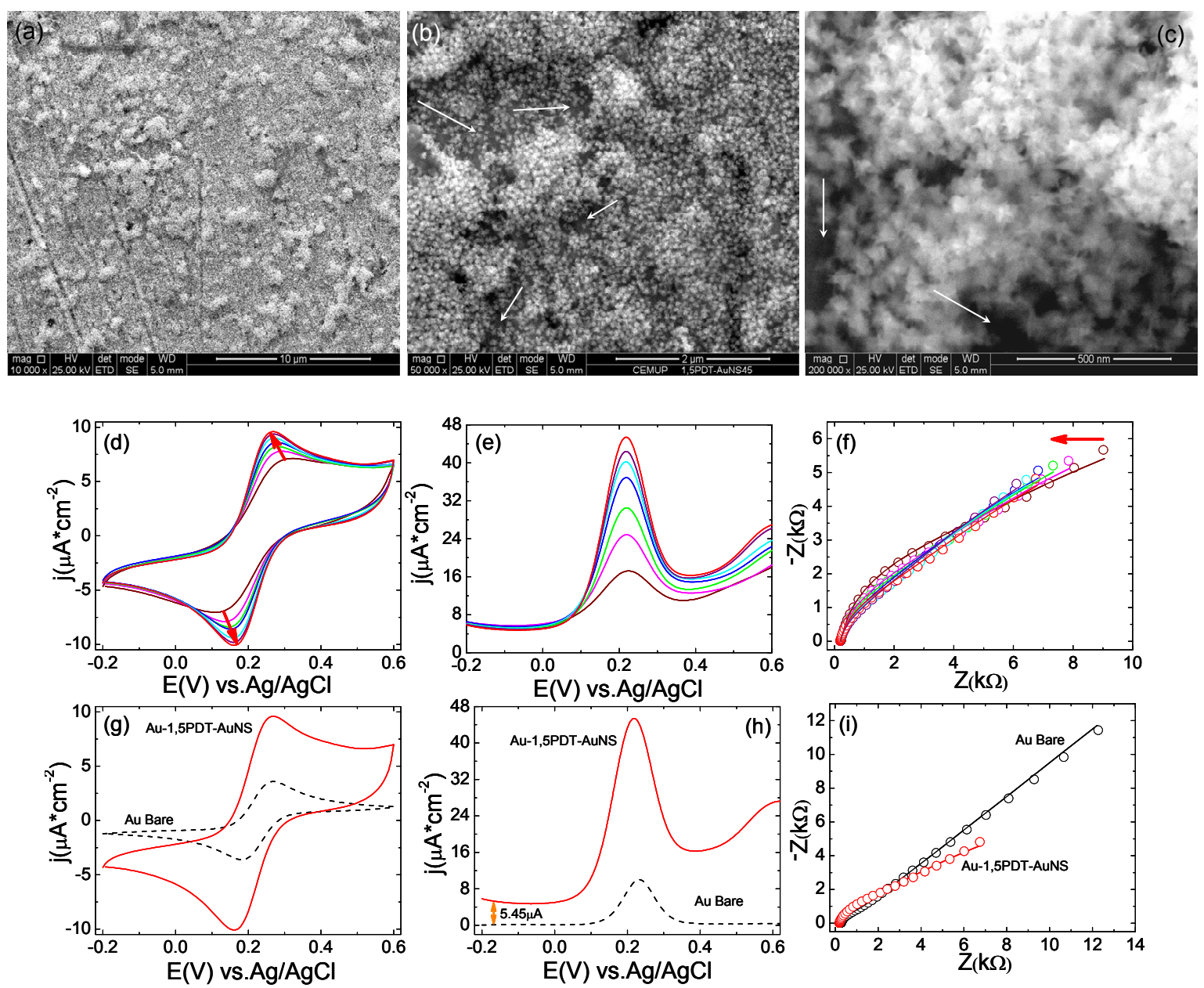

The physical aspect of the Au-1,5PDT-AuNS modified electrode suggests that the 1,5PDT SAM was formed of disulfides layers as demonstrated by Qu et al. [9] and Chah et al. [11], a fact which may 
explain the different heights observed in the structure of the 1,5PDT-AuNS film (Figure 5a-c). The presence of a dense layer of AuNSs at the Au-1,5PDT-AuNS/aqueous solution interface had a strong influence on the electrochemical properties of the modified electrode. The electrochemical behavior observed previously (Figures 2 and 4) was confirmed, with a stronger effect of AuNSs in the overall electrochemical process. First of all, cyclic voltammograms with high current densities of $j_{\mathrm{a}}=9.60 \mu \mathrm{A} \mathrm{cm} \mathrm{cm}^{-2}(\Delta E \mathrm{p}=0.105 \mathrm{~V})$ were obtained by consecutive measurements at the $\mathrm{Au}-1,5 \mathrm{PDT}-\mathrm{AuNS}$ modified electrodes (Figure 5d, $24 \mathrm{~h}$ self-assembly time of AuNSs) in aqueous solution of $0.0005 \mathrm{M}\left[\mathrm{Fe}(\mathrm{CN})_{6}\right]^{4-/ 3-}$ and $0.1 \mathrm{M} \mathrm{NaClO}_{4}$. In the square wave voltammograms (Figure 5e), the maximum current densities were $45.4 \mu \mathrm{A} \mathrm{cm} \mathrm{c}^{-2}$ (at $0.216 \mathrm{~V}$ ), being 4.5 orders of magnitude higher than at bare Au electrode. The current densities in the SQWVs (Figure 5e) start at a slightly higher value $(5.45 \mu \mathrm{A})$ than at bare $\mathrm{Au}$ electrode (Figure $5 \mathrm{~h}$ ). This difference is most probably due to the increase of electrode area upon self-assembly of AuNSs. And last but not least, the impedance values have drastically decreased to half of the values of impedance recorded at bare gold electrode (see Nyquist plots represented in Figure 5i). It has to be noted that the solution resistance, Rs, has slightly decreased (Table 5) illustrating a drastic change of the dielectric properties of the electrodes surface upon self-assembly of AuNSs as a dense layer.

From the fittings of impedance spectra, it was confirmed that the area of the Au-1,5PDT-AuNS modified electrode $(24 \mathrm{~h})$ has increased due to 4.2 times increase of film capacitance, $C_{\mathrm{f}}$, as compared to the previous $\mathrm{Au}-1,5 \mathrm{PDT}-A u N S$ modified electrode $(16 \mathrm{~h})$ (compare final $C_{\mathrm{f}}$ values in Tables 3 and 5). The film resistance, $R_{\mathrm{f}}$, has decreased even more than at the previous AuNS modified electrodes implying that a higher number of tunneling channels have been created by increasing AuNSs surface density (lower starting $R_{\mathrm{f}}$ ) and additionally, the $R_{\mathrm{f}}$ was decreasing with consecutive measurements illustrating a progressive improvement of through-bond electron tunneling process (Table 5).

Table 5. Parameter values obtained from fittings of the EIS spectra represented in Figure $5 f$.

\begin{tabular}{cccccc}
\hline EIS plot & $\boldsymbol{R}_{\mathbf{S}}(\mathbf{k} \boldsymbol{\Omega})$ & $\boldsymbol{C}_{\mathbf{f}}(\boldsymbol{\mu} \mathbf{F})$ & $\boldsymbol{R}_{\mathbf{f}}(\mathbf{k} \boldsymbol{\Omega})$ & $\boldsymbol{C}_{\mathbf{d l}}(\boldsymbol{\mu} \mathbf{F})$ & $\boldsymbol{R}_{\mathbf{C T}}(\mathbf{k} \boldsymbol{\Omega})$ \\
\hline \multirow{2}{*}{ wine } & 0.223 & 11.7 & 0.96 & 6.79 & 1.94 \\
& $(1.0)$ & $(0.83)$ & $(0.75)$ & $(0.83)$ & $(0.78)$ \\
magenta & 0.214 & 11.5 & 0.67 & 7.19 & 1.28 \\
& $(0.98)$ & $(0.65)$ & $(0.90)$ & $(0.83)$ & $(0.67)$ \\
green & 0.214 & 12.4 & 0.57 & 8.34 & 0.77 \\
& $(0.90)$ & $(0.83)$ & $(0.87)$ & $(0.75)$ & $(0.72)$ \\
blue & 0.202 & 11.8 & 0.39 & 7.43 & 0.68 \\
& $(0.85)$ & $(0.75)$ & $(0.82)$ & $(0.92)$ & $(0.90)$ \\
cyan & 0.204 & 12.1 & 0.46 & 8.23 & 0.58 \\
& $(0.97)$ & $(0.86)$ & $(1.0)$ & $(0.98)$ & $(0.77)$ \\
purple & 0.202 & 11.03 & 0.56 & 8.20 & 0.62 \\
& $(1.05)$ & $(0.80)$ & $(0.76)$ & $(0.95)$ & $(0.82)$ \\
red & 0.205 & 14.4 & 0.63 & 13.3 & 0.43 \\
& $(0.88)$ & $(0.65)$ & $(0.91)$ & $(0.79)$ & $(0.87)$ \\
\hline
\end{tabular}

Moreover, the high efficiency of electron tunneling through the underlying molecules of 1,5PDT layer was confirmed by the continuous decrease of the electron tunneling coefficient, $\beta$, with 
consecutive electrochemical measurements (Table 6). The double layer capacitance, $C_{\mathrm{dl}}$, has progressively increased (Table 5) being seven times larger than at bare gold electrode. The $C_{\mathrm{dl}}$ increase confirms a strong charge accumulation at the surface of Au-1,5PDT-AuNS modified electrode $(24 \mathrm{~h})$.

Table 6. Heterogeneous electron transfer rate constants estimated based on Equations (1) and (2) and using $R_{\mathrm{CT}}$ parameters from Table 5 and the corresponding electron tunneling coefficients, respectively.

\begin{tabular}{ccc}
\hline EIS plot & $\boldsymbol{k}_{\text {et }}\left(\mathbf{c m ~ s}^{-\mathbf{1}}\right)$ & $\boldsymbol{\beta}\left(\AA^{-\mathbf{1}}\right)$ \\
\hline wine & $0.88 \times 10^{-5}$ & 0.93 \\
magenta & $1.33 \times 10^{-5}$ & 0.88 \\
green & $2.19 \times 10^{-5}$ & 0.83 \\
blue & $2.48 \times 10^{-5}$ & 0.81 \\
cyan & $2.91 \times 10^{-5}$ & 0.80 \\
purple & $2.72 \times 10^{-5}$ & 0.80 \\
red & $3.95 \times 10^{-5}$ & 0.76 \\
\hline
\end{tabular}

As previously discussed, this spontaneous charging effect was also observed at films containing larger AuNSs (with average core diameters of $63.50 \mathrm{~nm}$ and average tip lengths of $23.57 \mathrm{~nm}$ ) at low surface coverage on modified electrodes [31]. The apparent charge transfer resistance, $R_{\mathrm{CT}}$, has strongly decreased revealing a very efficient electron transfer process taking place between the redox probes in solution and the AuNS modified electrode (24 h, Table 5). Accordingly, the heterogeneous electron transfer rate constants have greatly increased (Table 6), the final value being 1.42 higher than at bare gold (compare $k_{\text {et }}$ values in Tables 2 and 6). The variations of all electrochemical parameters strongly suggest that the increased surface density of AuNS was improving the electrochemical process. Our work is consistent with the work of Diao et al. [29] which have also demonstrated that a higher particle density results in higher number of tunneling channels and then a smaller apparent tunneling resistance, $R_{\mathrm{t}}(\mathrm{app})$ determining a more reversible electrochemical process and higher current densities in the voltammograms.

\subsection{Electrochemistry of $\left[\mathrm{Fe}(\mathrm{CN})_{6}\right]^{4-/ 3-}$ at Au-1,5PDT-AuNS Modified Electrodes for AuNSs' Self-Assembly Time of $32 \mathrm{~h}$}

A final tested time of AuNSs' self-assembly was $32 \mathrm{~h}$. At this time of AuNSs' self-assembly, their coverage on Au-1,5PDT-SAM modified electrode was complete (Figure 6a-c) and the electrochemical properties of the bilayer modified electrode were reconfirmed (Figure $6 \mathrm{~d}-\mathrm{i}$ ). SEM analysis of the fourth AuNS modified electrode shows a compact structure of the bilayer (Figure $6 a-c$ ) very similar to the structure of the previous Au-1,5PDT-AuNS modified electrode (16 h, Figure 5a-c). Consecutive measured cyclic voltammograms in aqueous solution of $0.0005 \mathrm{M}\left[\mathrm{Fe}(\mathrm{CN})_{6}\right]^{4-/ 3-}$ redox probes at this fourth Au-1,5PDT-AuNS modified electrode show a strong increase of current densities with decreasing peak potential separations (Figure 6d). After eight measured CVs, the peak current densities were stabilized $\left(j_{\mathrm{a}}=17.4 \mu \mathrm{A} \mathrm{cm}{ }^{-2}, \Delta E \mathrm{p}=0.117 \mathrm{~V}\right.$ for red curve in Figure $\left.6 \mathrm{~d}, \mathrm{~g}\right)$. The peak-to peak potential separation of $\Delta E \mathrm{p}=0.117 \mathrm{~V}$ shows a less reversible electrochemical process than at 
previously modified electrodes suggesting that after $32 \mathrm{~h}$ self-assembly time for AuNS, the optimal surface coverage with AuNS is overpassed. Nevertheless, the overall electrochemical process shows interesting features. For example, continuous measurements of square wave voltammograms have revealed a progressive shift of peak currents toward less positive potentials and a dramatic increase of current densities demonstrating an excellent improvement of electron transfer process induced by the AuNSs present at the Au-1,5PDT-AuNS modified electrode/solution interface. During the electrochemical process, the formal redox potential in the SQWVs was shifted from $0.226 \mathrm{~V}$ to $0.224 \mathrm{~V}$, $0.220 \mathrm{~V}, 0.216 \mathrm{~V}, 0.210 \mathrm{~V}, 0.208 \mathrm{~V}$ and $0.184 \mathrm{~V}$ (wine to red plots in Figure 6e), the square wave voltammograms recorded at this AuNS modified electrode featuring also a second shoulder at about $0.016 \mathrm{~V}$ (green to dark cyan plots in Figure 6e) which was further shifted to $0.093 \mathrm{~V}$ (red plot, Figure 6e). The shift of peak potential position toward less positive potentials together with a strong increase of current densities in SQWVs (Figure 6e) illustrates a strong facilitation of the overall electron transfer process induced by the AuNSs. Accordingly, EIS measurements performed at the Au-1,5PDT-AuNS modified electrode $(32 \mathrm{~h})$ show a 4 times decrease of impedance values for all range of frequency (Figure 6f,i) as compared to bare gold. The solution resistance, Rs, has decreased to half of the initial value indicating dielectric changes of electrode surface.

From an electrochemical point of view, the charging of AuNS layer at the Au-1,5PDT-AuNS/solution interface has determined a decrease of solution resistance as presented in Table 7. The dielectric properties of the film have an obvious influence on the solution resistance, $R_{\mathrm{s}}$. As discussed previously, the dithiol layer determines an increase of solution resistance due to its hydrophobic nature. Contrarily, the AuNSs electrochemically charged and being attached in a high number at the surface of electrodes ( $24 \mathrm{~h}$ and $32 \mathrm{~h}$ self-assembly time, Figures 5 and 6 ), have formed very conductive layers, which have determined a decrease of solution resistance. The decrease of $R_{\mathrm{s}}$ was observed only at high surface coverage with AuNSs (Tables 5 and 7). At low surface coverage although the electrochemical charging of AuNSs was confirmed by the increase of $C_{\mathrm{dl}}$ parameter, the increased electrical conductivity of the AuNS layer is undetectable because no change in solution resistance was observed at Au-1,5PDT-AuNS modified electrodes with $8 \mathrm{~h}$ and $16 \mathrm{~h}$ AuNSs' self-assembly time (see $R_{\mathrm{S}}$ values in Tables 1 and 3). Additionally, a dramatic increase of film capacitance, as compared with the previous $C_{\mathrm{f}}$ values, illustrates a high increase of electrode area upon AuNSs self-assembly ( $32 \mathrm{~h}$, Table 7). The film capacitance was progressively increasing illustrating a swelling of the film structure upon penetration of the redox probes into the AuNS layer.

Overall, the resistance of the film $R_{\mathrm{f}}$ is very small indicating an extremely efficient tunneling process through the chain of the 1,5PDT-SAM toward the underlying bare gold surface. The double layer capacitance, $C_{\mathrm{dl}}$, has drastically and progressively increased illustrating a strong charging effect at the Au-1,5PDT-AuNS modified electrode caused by a continuous exchange of electrons between the AuNSs in the film and the redox probes in solution. Compared to $C_{\mathrm{dl}}$ estimated at bare gold electrode $(1.89 \mu \mathrm{F}$, Table 1$)$ the $C_{\mathrm{dl}}$ at this fourth Au-1,5PDT-AuNS modified electrode has increased 14 times $(27 \mu \mathrm{F}$, Table 7$)$. It is clearly demonstrated that AuNSs act as strong energy storage materials opening great possibilities for their use as "nanoelectrochemical" capacitors [35]. Even more interesting, a very surprising evolution of apparent charge transfer resistance, $\mathrm{R}_{\mathrm{CT}}$, is represented by its strong decrease almost to nullification (Table 7). The estimated heterogeneous electron transfer rate constants using $R_{\text {Ст }}$ parameters from Table 7 show a progressive improvement of electron transfer 
process in parallel with a strong electrical charging of AuNSs during consecutive electrochemical measurements.

Figure 6. (a-c) SEM images of gold nanostars covalently self-assembled on Au-1, 5PDT-SAM modified electrodes for $32 \mathrm{~h}$. Scale bars: $50 \mu \mathrm{m}$ (a), $3 \mu \mathrm{m}$ (b) and $500 \mathrm{~nm}$ (c); Evolution of cyclic (d) and square wave voltammetry (e) and EIS (f) measurements recorded at the Au-1,5PDT-AuNS modified electrode in aqueous solution of $0.0005 \mathrm{M}$ $\left[\mathrm{Fe}(\mathrm{CN})_{6}\right]^{4-/ 3-}$ and $0.1 \mathrm{M} \mathrm{NaClO}$. Comparison of final cyclic (g), square wave voltammograms (h) and EIS spectra (i) recorded in the same conditions at Au-1,5PDT-AuNS modified electrodes (red curves) and bare gold (black dashed curves). Scan rate $50 \mathrm{mV} / \mathrm{s}$. Frequency $10 \mathrm{~Hz}$.
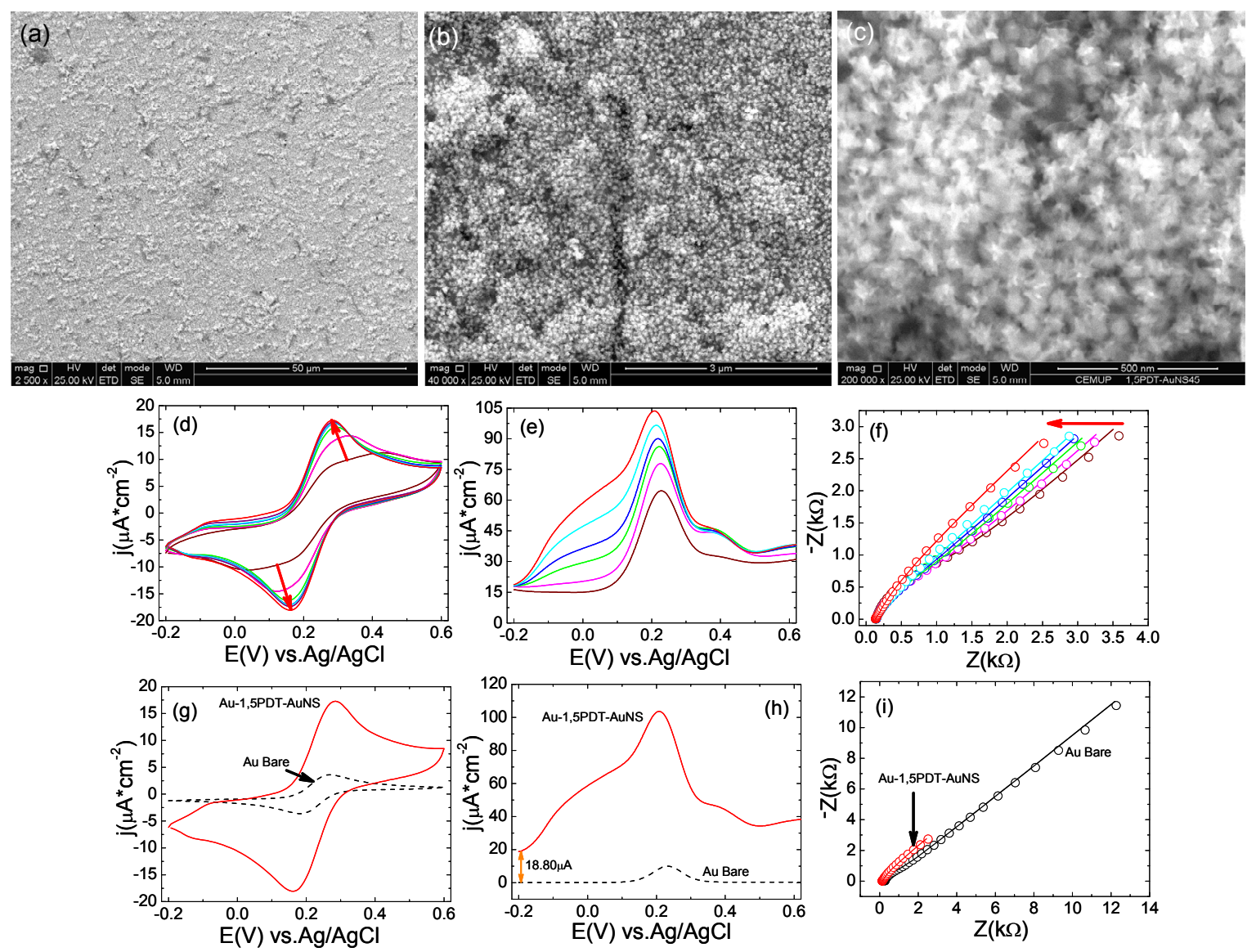

The $k_{\text {et }}$ values have drastically increased (Table 8) demonstrating that the electron transfer was becoming faster at Au-1,5PDT-AuNS modified electrode than at bare gold electrode. Moreover, the strong electrical charging of AuNSs has determined a high increase of $k_{\text {et }}$ values (Table 8). There is a strong jump of $k_{\text {et }}$ value from $10.77 \times 10^{-5} \mathrm{~cm} \cdot \mathrm{s}^{-1}$ to $85.0 \times 10^{-5} \mathrm{~cm} \cdot \mathrm{s}^{-1}$, this increase of $k_{\text {et }}$ suggesting an extremely high electrical charging of AuNSs within their layer. This strong charging of the AuNS layer had a strong influence on the electron tunneling process, which was highly improved, the electron tunneling coefficient decreasing continuously from $0.78 \AA^{-1}$ to $0.50 \AA^{-1}$ (Table 8).

In a report to be published, we will demonstrate that the increase of $k_{\text {et }}$ is possible due to the high number of electrons that could be stored on one single AuNS, the duration of charging process being 180 min for entire sample. During chemical charging with $\mathrm{NaBH}_{4}$, the most evident changes were 
observed at the tips of gold nanostar. The surface plasmon band of tips was blue shifted whereas its absorbance was increased revealing clearly a charging process. Moreover, their discharging process was very slow taking days to be completed. Compared to dense gold nanorod films at which the fastest electron transfer was depicted through a $k_{\mathrm{et}}$ of $1.58 \times 10^{-5} \mathrm{~cm} \cdot \mathrm{s}^{-1}$, the dense AuNSs films have generated clearly higher $k_{\text {et }}$ values: $3.95 \times 10^{-5} \mathrm{~cm} \cdot \mathrm{s}^{-1}(24 \mathrm{~h})$ and $85.0 \times 10^{-5} \mathrm{~cm} \cdot \mathrm{s}^{-1}(32 \mathrm{~h})$ respectively. These results have clearly demonstrated that AuNSs have a higher electrocatalytic effect combined with excellent energy storage abilities.

Table 7. Parameter values obtained from fittings of the impedance data represented in Figure $6 f$.

\begin{tabular}{cccccc}
\hline EIS plot & $\boldsymbol{R}_{\mathbf{s}}(\mathbf{k} \boldsymbol{\Omega})$ & $\boldsymbol{C}_{\mathbf{f}}(\boldsymbol{\mu F})$ & $\boldsymbol{R}_{\mathbf{f}}(\mathbf{k} \boldsymbol{\Omega})$ & $\boldsymbol{C}_{\mathrm{dl}}(\boldsymbol{\mu} \mathbf{F})$ & $\boldsymbol{R}_{\mathrm{CT}}(\mathbf{k} \boldsymbol{\Omega})$ \\
\hline \multirow{2}{*}{ wine } & 0.15 & 20.2 & 0.40 & 15.2 & 0.51 \\
& $(0.76)$ & $(0.86)$ & $(0.82)$ & $(0.50)$ & $(0.92)$ \\
magenta & 0.15 & 20.0 & 0.27 & 17.5 & 0.44 \\
& $(0.72)$ & $(0.79)$ & $(0.96)$ & $(0.92)$ & $(0.68)$ \\
green & 0.15 & 21.3 & 0.28 & 18.1 & 0.40 \\
& $(0.77)$ & $(0.83)$ & $(0.96)$ & $(0.59)$ & $(0.71)$ \\
blue & 0.15 & 23.1 & 0.26 & 26.3 & 0.35 \\
& $(1.08)$ & $(0.95)$ & $(0.94)$ & $(0.69)$ & $(0.76)$ \\
cyan & 0.145 & 24.8 & 0.17 & 26.0 & 0.16 \\
& $(0.81)$ & $(0.76)$ & $(0.71)$ & $(0.58)$ & $(0.68)$ \\
red & 0.14 & 26.5 & 0.11 & 27.0 & 0.02 \\
& $(0.67)$ & $(0.89)$ & $(0.87)$ & $(0.65)$ & $(0.80)$ \\
\hline
\end{tabular}

Table 8. Heterogeneous electron transfer rate constants estimated based on $\mathrm{R}_{\mathrm{CT}}$ parameters from Table 7.

\begin{tabular}{ccc}
\hline EIS plot & $\boldsymbol{k}_{\text {et }}\left(\mathbf{c m ~ s}^{-\mathbf{1}}\right)$ & $\boldsymbol{\beta}\left(\boldsymbol{\AA}^{-\mathbf{1}}\right)$ \\
\hline wine & $3.31 \times 10^{-5}$ & 0.78 \\
pink & $3.90 \times 10^{-5}$ & 0.76 \\
green & $4.30 \times 10^{-5}$ & 0.75 \\
blue & $4.85 \times 10^{-5}$ & 0.74 \\
cyan & $10.77 \times 10^{-5}$ & 0.65 \\
red & $85.0 \times 10^{-5}$ & 0.50 \\
\hline
\end{tabular}

\section{Experimental Section}

\subsection{Materials and Methods}

\subsubsection{Chemicals}

Hydrogen tetrachloroaurate (III) trihydrate $\left(\mathrm{HAuCl}_{4} \cdot 3 \mathrm{H}_{2} \mathrm{O}, 99.999 \%\right.$, Sigma Aldrich, Steinheim, Germany), polyvinylpyrrolidone (Sigma Aldrich, analytical grade, MW 10,000), sodium borohydride $\left(\mathrm{NaBH}_{4}, 96 \%\right.$, Sigma Aldrich, Sintra, Portugal), $N, N$-dimethylformamide (DMF, $\geq 99.8 \%$, Merck, Steinheim, Germany) 1,5 pentanedithiol, (Aldrich, 96\%, Sintra, Portugal), potassium hexacyanoferrate (III), potassium hexacyanoferrate (II) (pa quality) and $\mathrm{NaClO}_{4}$ (pa quality), $\mathrm{H}_{2} \mathrm{SO}_{4}$ (pure, Pronalab), 
$\mathrm{H}_{2} \mathrm{O}_{2} 30 \%$ (Fluka, Steinheim, Germany) were used as received. Millipore filtered water (resistivity > $18 \mathrm{M} \Omega \cdot \mathrm{cm}$ ) was used for preparation of all aqueous solutions. Prior use, all glassware were cleaned with freshly prepared aqua regia $\left(\mathrm{HNO}_{3}: \mathrm{HCl}=1: 3 \% v / v\right)$, rinsed abundantly with Millipore water and dried.

\subsubsection{Synthesis of Gold Seeds}

Spherical gold nanoparticles of $15 \mathrm{~nm}$ were prepared by standard citrate reduction [36-38]. Briefly, $5 \mathrm{~mL}$ of $1 \mathrm{wt} \%$ sodium citrate aqueous solution was added under continuous stirring to a boiling aqueous solution of $\mathrm{HAuCl}_{4}(100 \mathrm{~mL}, 0.5 \mathrm{mM})$ and allowed to react for $15 \mathrm{~min}$. The Erlenmeyer with the resulted $\mathrm{Au}$ seeds solution was removed from the heating plate and the solution was let to cool at room temperature. Consecutively, the $\mathrm{Au}$ particles were transferred into ethanol through PVP modification. Thus, $5 \mathrm{~mL}$ of a PVP (MW 10,000) aqueous solution, containing $0.2 \mathrm{~g}$ of PVP was added dropwise to the $\mathrm{Au}$ colloid and allowed to react overnight. The final solution was centrifuged at $4000 \mathrm{rpm}$ for $90 \mathrm{~min}$, the supernatant removed and the spherical nanoparticles redispersed in ethanol.

\subsubsection{Synthesis of Gold Nanostars}

The gold nanostars were synthesized as follows: $82 \mu \mathrm{L}$ of an aqueous solution of $50 \mathrm{mM} \mathrm{HAuCl}_{4}$ was mixed with $15 \mathrm{~mL}$ of $10 \mathrm{mM}$ PVP (MW 10,000) solution in DMF [18]. After the complete disappearance of the $\mathrm{Au}^{3+}$ CTTS absorption band at $325 \mathrm{~nm}$, a certain amount of preformed-seed dispersion was added under continuous stirring and allowed to react until completion of the reaction (no further changes in the UV-Vis-NIR spectra). For this specific synthesis, a ratio [HAuCl 4 ] to [seed] of 45 was used. The gold nanostars (AuNSs) were purified by washing with absolute ethanol and Millipore water through repeated steps of centrifugation and removal of supernatant. One washing step with $\mathrm{H}_{2} \mathrm{SO}_{4}: \mathrm{H}_{2} \mathrm{O}_{2}$ (1:1) mixture was performed for removal of PVP [25], followed by abundant washing with Millipore water. Finally, the gold nanostars were redispersed in $15 \mathrm{~mL}$ of Millipore water.

\subsubsection{Characterization of Gold Nanostars}

A drop of aqueous solution containing AuNSs was placed on formvar-carbon coated copper grids and naturally dried in air prior characterization by transmission electron microscopy. A JEOL JEM 1010 transmission electron microscope (TEM) operating at an acceleration voltage of $100 \mathrm{kV}$ was used for particle size analysis and low magnification imaging. Optical characterization of gold nanostars was carried out by UV-Vis-NIR spectroscopy using a Hitachi U-3000 spectrophotometer.

\subsubsection{Fabrication of Gold Nanostar Films}

First, gold electrodes of $0.0314 \mathrm{~cm}^{2}$ area were cleaned by repeated soaking in piranha solution $(3: 1(v / v)$ concentrated $\mathrm{H}_{2} \mathrm{SO}_{4}: \mathrm{H}_{2} \mathrm{O}_{2}, 30 \%$, Caution! Piranha solution is corrosive and reacts violently with organic materials) and washing with Millipore water, polished on micro cloth pads (Buehler) using polycrystalline diamond suspension of 1 and $0.25 \mu \mathrm{m}$ and sonicated in Millipore water for $20 \mathrm{~min}$. A final step was electrochemical cleaning by cyclic voltammetry in a $0.02 \mathrm{M}$ aqueous solution of $\mathrm{HClO}_{4}$, for a potential window of -0.4 to $1.5 \mathrm{~V}$ at a scan rate of $0.1 \mathrm{~V} / \mathrm{s} v s$. $\mathrm{Ag} / \mathrm{AgCl} / \mathrm{KCl}(3 \mathrm{M})$. The clean 
bare $\mathrm{Au}$ electrodes were immersed in $0.015 \mathrm{M}$ ethanolic solution of 1,5pentanedithiol (1,5PDT) for $24 \mathrm{~h}$ at room temperature. The 1,5pentanedithiol modified electrodes (Au-1,5PDT-SAM) were washed with absolute ethanol and consecutively immersed in aqueous solution of gold nanostars $(1 \mathrm{mg} / \mathrm{mL})$ for $8 \mathrm{~h}, 16 \mathrm{~h}, 24 \mathrm{~h}$ and $32 \mathrm{~h}$. These electrodes were used as working electrodes in all electrochemical measurements.

\subsubsection{Characterization of Gold Nanostar Films}

The surface loadings of gold nanostar on the Au-1,5PDT-SAM modified electrodes, as a function of their self-assembly time, were analyzed by High resolution (Schottky, Eindhoven, The Netherlands) Environmental Scanning Electron Microscope (FEI Quanta 400FEG /EDAX Genesis X4M, Eindhoven, The Netherlands). The electrochemical properties of the Au-1,5PDT-AuNS modified electrodes were studied by cyclic voltammetry, square wave voltammetry and electrochemical impedance spectroscopy. All electrochemical measurements were performed on a PGSTAT 302N potentiostat (EcoChemie B.V., Utrech, The Netherlands) in a standard three-electrode single compartment cell. A platinum wire and an $\mathrm{Ag} / \mathrm{AgCl}(3 \mathrm{M} \mathrm{KCl})$ electrode were used as counter and reference electrodes, respectively. The electrolyte solution consisted of $0.0005 \mathrm{M}\left[\mathrm{Fe}(\mathrm{CN})_{6}\right]^{4-}, 0.0005 \mathrm{M}\left[\mathrm{Fe}(\mathrm{CN})_{6}\right]^{3-}$ and $0.1 \mathrm{M} \mathrm{NaClO}_{4}$.

\section{Conclusions}

It was demonstrated that gold nanostars of $70 \mathrm{~nm}$ tip to tip distance could be excellent electrocatalysts, if covalently self-assembled as dense layers on 1,5pentanedithiol modified electrodes. The electron transfer process was studied at Au-1,5PDT-AuNS modified electrodes as a function of AuNS surface coverage in aqueous solution of $\left[\mathrm{Fe}(\mathrm{CN})_{6}\right]^{4-/ 3-}$ redox probes. At low surface coverage, the electron transfer was slower than at bare gold electrode. At dense layers of AuNSs a strong improvement of electron transfer process together with an efficient electron tunneling process through the aliphatic chains of the 1,5PDT layer was illustrated by progressive decrease of both charge transfer resistance, $R_{\mathrm{CT}}$, and tunneling resistance $R_{\mathrm{f}}$. Additionally, strong charging effects were revealed for the first time by a drastic increase of double layer capacitance, $C_{\mathrm{dl}}$. Overall, the electrochemical properties of AuNS have revealed a complex and interesting behavior which makes them great candidates for fabrication of energy storage devices, electrochemical sensors or biosensors.

\section{Acknowledgments}

Financial support from Fundacão para a Ciência e a Tecnologia (FCT) of Portugal through the fellowship SFRH/BPD/39294/2007 is gratefully acknowledged. Rui Rocha is gratefully acknowledged for SEM measurements at CEMUP in Porto.

\section{Conflict of Interest}

The authors declare no conflict of interest. 


\section{References}

1. Talapin, D.V.; Lee, J.-S.; Kovalenko, M.K.; Shevchenko, E.V. Prospects of colloidal nanocrystals for electronic and optoelectronic applications. Chem. Rev. 2010, 110, 389-458.

2. Andres, R.P.; Bein, T.; Dorogi, M.; Feng, S.; Henderson, J.I.; Kubiak, C.P.; Mahoney, W.; Osifchin, R.G.; Reifenberger, R. Coulomb staircase at room temperature in a self-assembled molecular nanostructure. Science 1996, 272, 1323-1325.

3. Chirea, M.; Cruz, A.; Pereira, C.M.; Silva, A.F. Size-dependent electrochemical properties of gold nanorods. J. Phys. Chem. C 2009, 113, 13077-13087.

4. Chirea, M.; Borges, J.; Pereira, C.M.; Silva, A.F. Density-dependent electrochemical properties of vertically aligned gold nanorods. J. Phys. Chem. C 2010, 114, 9478-9488.

5. Shein, J.B.; Lai, L.M.H.; Eggers, P.K.; Paddon-Row, M.N.; Gooding, J.J. Formation of efficient electron transfer pathways by adsorbing gold nanoparticles to self-assembled monolayer modified electrodes. Langmuir 2009, 25, 11121-11128.

6. Gabriela, P.; Kissling, G.P.; Miles, D.O.; Fermín, D.J. Electrochemical charge transfer mediated by metal nanoparticles and quantum dots. Phys. Chem. Chem. Phys. 2011, 13, 21175-21185.

7. Ashur, I.; Schulz, O.; McIntosh, C.L.; Pinkas, I.; Ros, R.; Jones, A.K. Transparent gold as a platform for adsorbed Protein Spectroelectrochemistry: Investigation of cytocrom c and azurin. Langmuir 2012, 28, 5861-5871.

8. De Boer, B.; Frank, M.M.; Chabal, Y.J.; Jiang, W.; Eric Garfunkel , E.; Bao, Z. Metallic contact formation for molecular electronics: interactions between vapor-deposited metals and self-assembled monolayers of Conjugated mono- and dithiols. Langmuir 2004, 20, 1539-1542.

9. Qu, D.; Kim, B.-C.; Lee, C.-W.J.; Ito, M.; Noguchi, H.; Uosaki, K. 1,6-Hexanedithiol self-assembled monolayers on $\mathrm{Au}(111)$ investigated by electrochemical, spectroscopic, and molecular mechanics methods. J. Phys. Chem. C 2010, 114, 497-505.

10. Akkerman, H.B.; Kronemeijer, A.J.; van Hal, P.A.; de Leeuw, D.M.; Blom, P.W.M.; de Boer, B. Self-assembled-monolayer formation of long alkanedithiols in molecular junctions. Small 2008, 4 , $100-104$.

11. Chah, S.; Fendler, J.H.; Yi, J. In-situ analysis of step-wise self-assembled 1,6Hexanedithiol multilayers by surface plasmon resonance measurements. Chem. Commun. 2002, 2094-2095, doi:10.1039/B206817F.

12. Reed, M.A.; Zhou, C.; Muller, C.J.; Burgin, T.P.; Tour, J.M. Conductance of a molecular junction. Science 1997, 278, 252-254.

13. Cui, X.D.; Primak, A.; Zarate, X.; Tomfohr, J.; Sankey, O.F.; Moore, A.L.; Moore, T.A.; Gust, D.; Harris, G.; Lindsay, S.M. Reproducible measurements of single molecule conductivity. Science 2001, 294, 571-574.

14. Yang, Y.-C.; Lee, Y.-L.; Yang, L.-Y.O.; Yau, S.-Y. In situ scanning tunneling microscopy of 1,6-hexanedithiol, 1,9-nonanedithiol, 1,2-benzenedithiol, and 1,3-benzenedithiol adsorbed on Pt (111) electrodes. Langmuir 2006, 22, 5189-5195.

15. Niskala, J.R.; Rice, W.C.; Bruce, R.C.; Merkel, T.J.; Tsui, F.; You, W. Tunneling characteristics of Au-alkanedithiol-Au junctions formed via nanotransfer printing (nTP). J. Am. Chem. Soc. 2012, 134, 12072-12082. 
16. Lu, M.; Li, X.H.; Yu, B.Z.; Li, H.L. Electrochemical behavior of Au colloidal electrode through layer-by-layer self-assembly. J. Colloid Interface Sci. 2002, 248, 376-382.

17. Qu, D.; Uosaki, K. Electrochemical metal deposition on top of an organic monolayer. J. Phys. Chem. B 2006, 110, 17570-17577.

18. Barbosa, S.; Agrawal, A.; Rodríguez-Lorenzo, L.; Pastoriza-Santos, I.; Alvarez-Puebla, A.R.; Kornowski, A.; Weller, H.; Liz-Marzán, L.-L. Tuning size and sensing properties in colloidal gold nanostars. Langmuir 2010, 26, 14943-14950.

19. Khoury, C.G.; Vo-Dinh, T. Gold nanostars for surface-enhanced Raman scattering: synthesis, characterization and optimization. J. Phys. Chem. C 2008, 112, 18849-18859.

20. Kumar, P.S.; Pastoriza-Santos, I.; Rodríguez-González, B.; de Abajo, F.J.G.; Liz-Marzán, L.M. High-yield synthesis and optical response of gold nanostars. Nanothechnology 2008, 19, 015606.

21. Rodríguez-Lorenzo, L.; Alvarez-Puebla, R.A.; Pastoriza-Santos, I.; Mazzucco, S.; Stéphan, O.; Kociak, M.; Liz-Marzán, L.M.; de Abajo, F.J.G. Zeptomol detection through controlled ultrasensitive surface-enhanced Raman scattering. J. Am. Chem. Soc. 2009, 131, 4616-4618.

22. Rodríguez-Lorenzo, L.; Alvarez-Puebla, R.A.; de Abajo, F.J.G.; Liz-Marzan, L.L. Surface enhanced Raman scattering using star-shaped gold colloidal nanoparticles. J. Phys. Chem. C 2010, 114, 7336-7340.

23. Shao, L.; Susha, A.S.; Cheung, L.S.; Sau, T.K.; Rogach, A.L.; Wang, J. Plasmonic properties of single multispiked gold nanostars: Correlating modeling with experiments. Langmuir 2012, 28 , 8979-8984.

24. Dondapati, S.K.; Sau, T.K.; Hrelescu, C.; Klar, T.A.; Stefani, F.D.; Feldmann, J. Label-free biosensing based on single gold nanostars as plasmonic transducers. ACS Nano 2010, 4, 6318-6322.

25. Monzo, J.; Kopper, M.T.M.; Rodriguez, P. Removing polyvinylpyrrolidone from catalytic Pt nanoparticles withouth modification of superficial order. Chem. Phys. Chem. 2012, 13, 709-715.

26. Chirea, M.; Garcia-Morales, V.; Manzanares, J.A.; Pereira, C.; Gulaboski, R.; Silva, F. Electrochemical characterization of polyelectrolyte/gold nanoparticle multilayers self-assembled on gold electrodes. J. Phys. Chem. B 2005, 109, 21808-21817.

27. Chirea, M.; Pereira, C.; Silva, F. Catalytic effect of gold nanoparticles self-assembled in multilayered polyelectrolyte films. J. Phys. Chem. C 2007, 111, 9255-9266.

28. Diao, P.; Liu, Z. Electrochemistry at chemically assembled single-wall carbon nanotube arrays. J. Phys. Chem. B 2005, 109, 20906-20913.

29. Diao, P.; Guo, M.; Zhang, Q. How does the particle density affect the electrochemical behaviour of gold nanoparticle assembly? J. Phys. Chem. C 2008, 112, 7036-7046.

30. Finklea, H.O.; Hanshew, D.D. Electron-transfer kinetics in organized thiol monolayers with attached pentaammine(pyridine)ruthenium redox centers. J. Am. Chem. Soc. 1992, 114, 3173-3181.

31. Chirea, M.; Pereira, C.; Silva, F. Shape dependence of electron transfer kinetics at nanometer sized films. Lett. Appl. Nano Biosci. 2012, 1, 56-62.

32. Rodriguez-Lorenzo, L.; Romo-Herrera, J.M.; Perez-Juste, J.; Alvarez-Puebla, R.A.; Liz-Marzan, L.M. Reshaping and LSPR tuning of Au nanostars in the presence of CTAB. J. Mater. Chem. 2011, 21, 11544-11549. 
33. Zou, R.; Zhang, Q.; Zhao, Q.; Peng, F.; Wang, H.; Yu, H.; Yang, J. Thermal stability of gold nanorods in an aqueous solution. Colloids Surf. A 2010, 372, 177-181.

34. Zhang, L.; Holt, C.M.B.; Luber, E.J.; Olsen, B.C.; Wang, H.; Danaie, M.; Cui, X.; Tan, X.; Lui, W.V.; Kalisvaart, W.P.; et al. High rate electrochemical capacitors from three-dimensional arrays of vanadium nitride functionalized carbon nanotubes. J. Phys. Chem. C 2011, 115, 24381-24393.

35. Kötza, R.; Carlen, M. Principles and applications of electrochemical capacitors. Electrochim. Acta 2000, 45, 2483-2498.

36. Graf, C.; Vossen, L.J.; Imhof, A.; van Blaaderen, A. A general method to coat colloidal particles with silica. Langmuir 2003, 19, 6693-6700.

37. Turkevich, J.; Stevenson, P.C.; Hillier, J. A study of the nucleation and growth processes in the synthesis of colloidal gold. Discuss. Faraday Soc. 1951, 11, 55-75.

38. Frens, G. Controlled nucleation for the regulation of the particle size in monodisperse gold suspensions. Nature 1973, 241, 20-22.

(C) 2013 by the authors; licensee MDPI, Basel, Switzerland. This article is an open access article distributed under the terms and conditions of the Creative Commons Attribution license (http://creativecommons.org/licenses/by/3.0/). 